\title{
Evaluación de los autocuidados del pie en pacientes diabéticos. Análisis retrospectivo (2008-2013)
}

\author{
Evaluation foot self care in diabetic patients. \\ Retrospective Analysis (2008-2013)
}

\section{Emmanuel Navarro-Flores ${ }^{1}$, Gabriel Gijón-Noguerón², José Antonio Cervera-Marín², María Teresa LabaJos-Manzanares ${ }^{3}$}

\author{
${ }^{1}$ Máster en Investigación en Cuidados de Salud. Departamento de Enfermería y Podología. Universidad de Málaga. \\ emmnavflo@uma.es \\ ${ }^{2}$ Doctor. Departamento de Enfermería y Podología. Universidad de Málaga. \\ gagijon@uma.es \\ jacervera@uma.es \\ ${ }^{3}$ Doctor Departamento de Psiquiatría y Fisioterapia. Universidad de Málaga. \\ mtlabajos@uma.es
}

\author{
Correspondencia: \\ Emmanuel Navarro Flores \\ Facultad de Ciencias de la Salud \\ Paseo Martiricos S/N \\ E-29011 Málaga \\ Correo electrónico: emmvaflo@uma.es
}

\begin{abstract}
RESUMEN
El objetivo es evaluar el estado en el que se encuentra la relación del autocuidado y autoexploración de los pies con respecto al desarrollo de complicaciones de diabetes mellitus.

Mediante revisión sistemática de los estudios publicados relativos a la evaluación de los hábitos de salud en cuanto a intervenciones de educación para la salud sobre pie diabético, así como ensayos clínicos, metaanálisis y revisiones Cocrhane. Durante el periodo comprendido entre 2000 y marzo de 2013

De los estudios identificados solamente 33 fueron seleccionados para el desarrollo de nuestra investigación de los cuales habían sido seleccionados previamente 34 de pubmed, 12 de web of knowledge y 4 de Cochrane que fueron considerados como válidos al cumplir el requisito de tratarse de intervenciones que reducían las complicaciones en los pies derivadas de la diabetes.

Las estrategias orientadas al cambio de comportamiento tienen efectividad sobre el control metabólico de la enfermedad y la reducción de amputaciones. Pero es necesario validar una herramienta fiable que permita conocer el estado del desarrollo de conductas saludables y que éstas se mantengan en el tiempo, dado el déficit metodológico que presentan la mayoría de los estudios, en cuanto a selección de la muestra y tiempo de estudio.

Palabras clave: revisión sistemática; diabetes; pie diabético; autocuidado; calidad de vida.
\end{abstract}

\begin{abstract}
Purpose: The purpose of this study is to analyze other studies and research on foot self care and self-examination in patients with diabetes, taken from different sources of the scientific literature. Methods: The authors carried out a systematic review of published studies (including clinical trials, meta-analysis and Cochrane reviews), about the evaluation of health habits and health education concerning foot care in patients with diabetes. The authors researched articles using the terms "diabetic foot self-care, diabetic foot self-management, diabetes self-care and diabetes selfmanagement" in the Medline, Web of Science, Cochrane Database, CINAHL, Scopus and EMBASE databases. Only 33 of the studies found here and published between January 1990 and May 2012 fulfilled the requirements, as they dealt with medical procedures to reduce the complications in feet caused by diabetes. Results: Of a total of 479 studies identified, the authors previously selected 50, of which 34 were from Pubmed, 12 from Web of Knowledge and four from Cochrane. In the end, they only chose 33 in order to develop this research, of which three were on metaanalysis, four were systematic reviews, six were randomized clinical trials, three were observational studies and 17 were review articles. Conclusions: All the analyzed studies present strategies directed towards a change in behavior.
\end{abstract}


These strategies are effective regarding the metabolic control of the illness and the reduction of amputations. But it is necessary to validate a reliable tool to determine the development of healthy habits and to guarantee their continued practice, given that most studies present a lack of methodology regarding sample selection and time of study.

Key words: foot care; self care; self management; diabetic foot.

Referencia normalizada: Navarro-Flores, E., Gijón-Noguerón, G., Cervera-Marín, J.A., Labajos-Manzanares, M.T. Evaluación de los autocuidados del pie en pacientes diabéticos. Análisis retrospectivo (2008-2013). Rev. Int. Cienc. Podol. 2014; 8(1): 25-35.

Sumario: 1. Introducción, 2. Material y método, 3. Resultados, 4. Discusión, 5. Limitaciones, 6. Conclusiones, Bibliografía. 


\section{INTRODUCCIÓN}

Se espera que la prevalencia mundial de diabetes aumente del 2,8\% del 2000 al 4,4\% en el año 2030, lo cual significa que 366 millones de personas se verán afectadas Las úlceras, una de las principales complicaciones de la diabetes, se presentan entre el $15 \%$ y $25 \%$ de los pacientes en algún momento de su vida. Así, las complicaciones relacionadas con el pie diabético suponen una grave carga, debido a su elevada incidencia ${ }^{1}$.

La diabetes mellitus (DM) en combinación con la presencia de úlceras, aumenta el riesgo de amputación, especialmente si están presentes signos de neuropatía, isquemia e infecciones profundas ${ }^{2}$. Por lo que, en este sentido, el autocuidado es fundamental en el manejo de la diabetes y la prevención de aparición de complicaciones de la misma.

El autocontrol de la diabetes por parte de los pacientes cumple, por tanto, el objetivo de ayudarles a tomar conciencia de su propia condición, mediante la mejora de sus conocimientos y habilidades para realizar acciones adecuadas de autocuidado y autoexploración, orientados hacia un cambio de comportamiento positivo, que les permita, en última instancia, reducir el riesgo de complicaciones ${ }^{2}$.

A pesar de la presencia de múltiples herramientas evaluadoras del estado de salud, existe una alta heterogeneidad entre las mismas. La calidad metodológica de estos instrumentos es de gran variabilidad, lo que limita la comparación o generalización de los resultados ${ }^{3}$.

En una revisión del año 2008 sobre autocontrol y educación para la diabetes, se sugería que los pacientes que no recibían información sobre cómo controlar la enfermedad, tenían un notable riesgo de poder desarrollar complicaciones de pie diabético ${ }^{4}$, en concreto cuatro veces superior, con respecto a aquellos pacientes que recibían información sobre el autocontrol de la enfermedad. En este sentido, recordemos que uno de los objetivos de la declaración de Saint Vincent en cuanto a reducción de amputaciones se basaba en la estrategia de la educación como herramienta preventiva ${ }^{5}$.

De hecho, algunas guías internacionales, como la de la Asociación Americana de Diabetes (ADA), respaldaron este planteamiento, mediante el empleo de estrategias de prevención de úlceras por medio de la optimización del control metabólico, la identificación de pacientes que presentan alto riesgo de ulceración y la educación del paciente para promover el autocuidado de los pies, motivo por el cual se justifica el presente trabajo ${ }^{6}$.

Diversos estudios concluyen que la educación del paciente, puede mejorar los resultados de forma directa, en cuanto a disminución de complicaciones derivadas de la diabetes. Sirviendo de ejemplo la atención podológica, mediante la evaluación del riesgo de ulceración de los pies y la orientación motivacional para reforzar las conductas de autocuidado de los pies $^{7}$. Un factor determinante para el cumplimiento de las recomendaciones de autocuidado es el estilo de asesoramiento médico.

En la actualidad, la educación en el cuidado de los pies se dirige, en gran parte, a aquellos pacientes con antecedentes de complicaciones, sobre todo los que presentan aumento de los niveles de HbA1c y los diabéticos de varios años de evolución ${ }^{8}$.

Sin embargo, los pacientes cuyo perfil se califica de bajo riesgo, pueden desarrollar complicaciones con relativa rapidez en ausencia de un buen control glucémico y prácticas de autocuidado, que puedan facilitar la rápida identificación de cambios en la sensibilidad ${ }^{9}$.

De este modo, los últimos estudios han hallado que los pacientes con neuropatía no mostraron mayor presencia de deformidades que los que no eran diabéticos neuropáticos. Por lo que la presencia de neuropatía y ulceras, que siempre ha estado asociada a la presencia de prominencias óseas, queda en entredicho ${ }^{10}$.

El propósito de este trabajo es ofrecer una revisión actualizada de actuaciones de los diferentes criterios de autocuidados que utilizan los diabéticos para sus pies.

\section{MATERIAL Y MÉTODO}

Para tratar el tema, se realizó revisión bibliográfica de la literatura médica para identificar los posibles estudios existentes. Tras realizar búsqueda bibliográfica en las bases de datos Pubmed, CINAHL, Scopus, EMBASE, Web of Knowledge, y Cocrhane Library durante el pe- 
riodo comprendido entre enero de 2008 y marzo de 2013 mediante la utilización los términos "diabetic, foot, self-care", utilizando las combinaciones y las variaciones de los términos de búsqueda siguientes diabetes and selfmanagement, diabetes and self-care, diabetic foot and selfmanagement, tras cribar y estimar, como de mayor relevancia, seleccionamos los artículos más oportunos para la elaboración del presente trabajo y que se muestran en la Figura 1:

\section{Tipos de estudios}

Ensayos controlados aleatorizados (ECA) y pseudo-aletorizados, ensayos clínicos casocontrol (ECC) (utilizando métodos de asignación a los participantes que no son estrictamente aleatorios) y aquellos que compararon las intervenciones educativas versus ninguna intervención (control) o para otras intervenciones en el pie diabético.

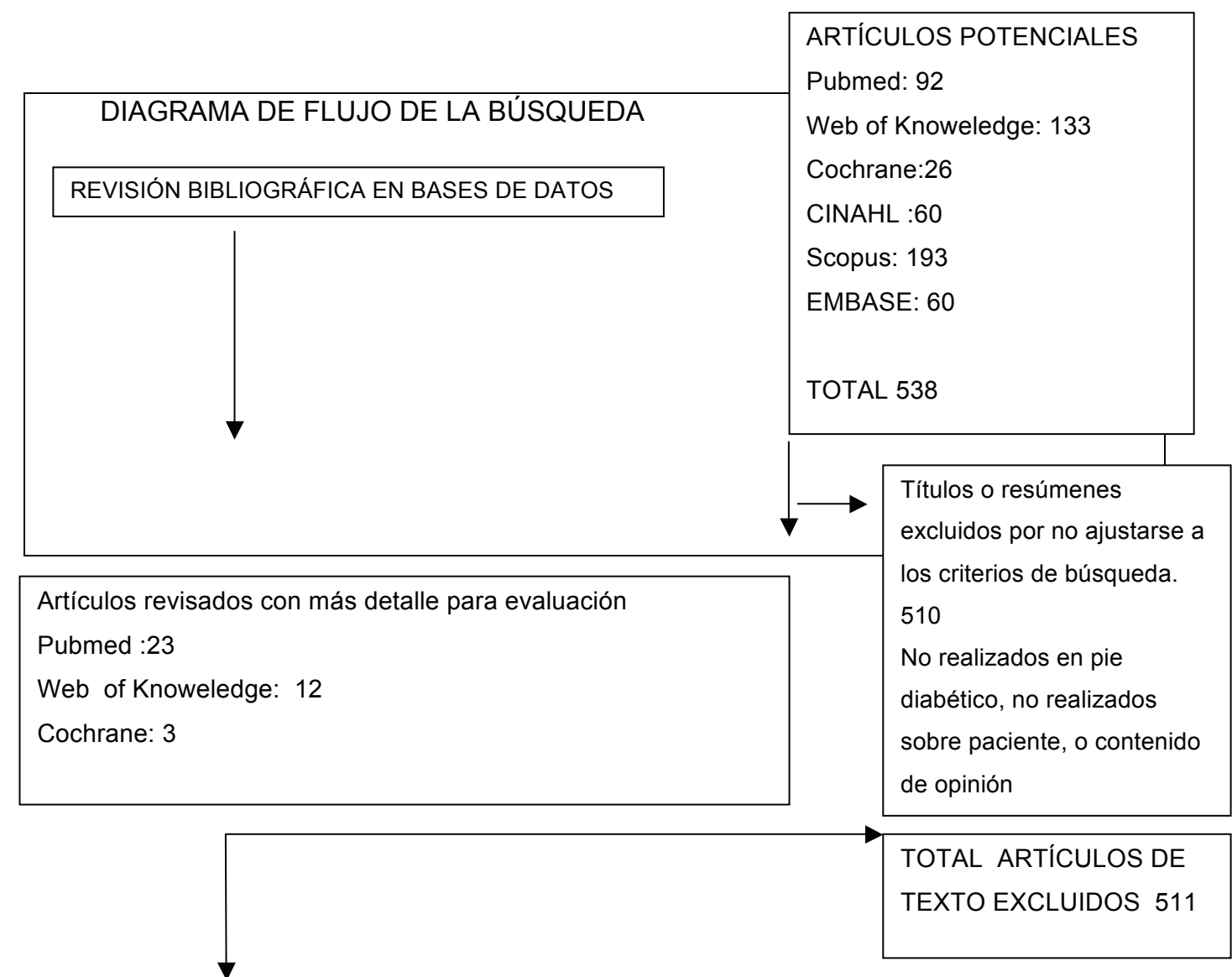

ARTICULOS INCLUIDOS EN EL ESTUDIO

Pubmed: 13

Web of Knoweledge: 12

Cochrane: 2

Figura 1. Diagrama de flujo de búsqueda 


\section{Tipos de participantes}

Dado que no existe una definición universalmente aceptada para el cuidado del pie diabético, se siguió utilizando las recomendaciones aceptadas en el documento de consenso internacional del grupo de investigación en pie diabético ${ }^{11}$.

Los estudios sobre la diabetes en general fueron elegibles para su inclusión, siempre que los resultados se presentasen por separado.

\section{Tipos de intervenciones}

En cuanto a las intervenciones se incluyeron las orientadas a la reducción de la presencia de úlceras o amputaciones. Pero se excluyeron los estudios que incluían algún tipo de intervención quirúrgica.

\section{Tipos de medidas de resultado}

\section{Resultados primarios}

- Autocuidado en pie diabético

- Autoexploración en pie diabético

\section{Resultados secundarios}

- Reducción de amputaciones

- Intervenciones educativas

- Medidas de calidad de vida

Los artículos con título o resumen inapropiado fueron excluidos de la búsqueda o bien después de haber sido leídos por completo, por las siguientes razones: no pertenecían a estudios realizados en pie diabético $(\mathrm{n}=176)$, no estaban relacionados con la reducción de riesgos $(n=182)$, la no intervención o intervención, no iba dirigidas a los pacientes $(\mathrm{n}=68)$, o el contenido era de tipo descriptivo $u$ opinión $(\mathrm{n}=39)$. Como resultado se incluyeron en estudio 34 artículos. De los cuales 12 estudios fueron de prevención primaria, cuatro versaban sobre la reducción de amputaciones, seis trataban la reaparición de úlceras 12 fueron sobre educación sanitaria y 2 sobre calidad de vida.

\section{Criterios de inclusión y exclusión}

Proceso de selección de estudios

Mediante la búsqueda por los términos que se refieren a "diabetes mellitus self care", "diabetes mellitus selfmanagement"; "diabetic foot self care" y "diabetic foot selfmanagement", y los procedimientos que se describen se obtienen los siguientes resultados:

Para la búsqueda de la Web of Knowledge, se utilizó el enlace de la Universidad de Málaga (http://jabega.uma.es/search*spi?/++ftlist/++ftl ist $/ 1,1,1, \mathrm{~B} / 1856 \sim \mathrm{b} 1415556 \& \mathrm{FF}=\& 1,0,1,0)$ por ser la base de datos más general. Tras leer el resumen completo de 133 artículos, 12 fueron relevantes para nuestra investigación.

Por lo que respecta a Medline se hizo la búsqueda en su página de Pubmed (http://www. ncbi.nlm.nih.gov/pubmed/). En ella se encontraron 92 artículos que se ajustaban a los criterios de búsqueda descritos, leyendo el resumen de 34, aunque sólo 23 fueron seleccionados como más relevantes para el desarrollo del presente trabajo.

La exclusión de algunos de los estudios se debe a que, una vez analizados, no resultaron útiles para nuestra investigación ya que no habían sido realizados sobre pacientes con pie diabético; su contenido era de opinión, o bien, la intervención no había sido dirigida a los pacientes.

También se realizó búsqueda de revisiones sistemáticas de Cocrhane donde se obtuvieron 26 resultados que se ajustaban al criterio de búsqueda establecido, pero sólo tres resultaron útiles para el objeto de estudio. Así como en otras bases de datos de referencia como son CINAHL, en la que se hallaron en total 60 resultados; Scopus con un total de 193 resultados válidos para el estudio $\mathrm{y}$, por último, se obtuvieron 10 para la búsqueda realizada en EMBASE. Estas búsquedas se efectuaron de igual modo y en los mismos términos que las realizadas en las bases anteriormente citadas.

Tratando de incluir los estudios de mayor calidad, los criterios de inclusión se limitaron a aquellos estudios que evaluaron la intervención de autocontrol de la diabetes que tenía por objetivo reducir los riesgos de complicación. Se excluyeron, también, los estudios publicados antes de 2008.

\section{RESULTADOS}

\section{Extracción de los datos}

Dos revisores de manera independiente realizaron la extracción de datos completa con una 
opinión uniforme de consenso. Por otra parte se construyó una herramienta de extracción de datos estandarizada con el fin de de identificar las características y detalles clave de cada estudio.

Los revisores de forma independiente realizaron un formulario con un pequeño subgrupo de estudios representativos para confirmar su contenido. Teniendo en cuenta las propiedades psicométricas, los detalles extraídos del estudio centrados en la descripción de la herramienta, objeto de medición, validez de contenido, validez de constructo, criterio de validez, sensibilidad y fiabilidad para los cuestionarios de salud, así como la descripción de instrumentos, medición de objetos, sensibilidad, especificidad y fiabilidad gold standard para las medidas objetivas de los artículos.

\section{Análisis de los datos}

Para cada estudio, se calculó el nivel de significación ( $\mathrm{p}$ - valor $<0.005$ ) y los intervalos de confianza (IC) al 95\% para los resultados continuos. Se planificó realizar metaanálisis, sin embargo hemos encontrado datos insuficientes.

\section{Evaluación de la calidad de los estudios}

Dos revisores independientes evaluaron la calidad de los estudios, y los desacuerdos se resolvieron por consenso. Los estudios se evaluaron críticamente mediante el Programa de habilidades en lectura crítica en español (Caspe) ${ }^{12}$.

En relación al autocuidado, hemos identificado siete revisiones sistemáticas y tres metaanálisis que se muestran en la Tabla 1, así como siete estudios primarios. Los estudios refieren sistemáticamente que la educación del paciente es un componente del cuidado de la diabetes. A partir de los citados metaanálisis se concluye que: los estudios de calidad inferior tienden a producir mayor efecto. Los principales resultados son que el conocimiento y desarrollo de la habilidad de autocuidado fue mejor en los programas de educación al paciente de mayor duración, y que los efectos del conocimiento y habilidades mejoran a largo plazo ${ }^{13}$.

Sin embargo, la mayoría de autores señalan que el conocimiento y las habilidades son necesarias, pero no suficientes, para asegurar un buen control de la diabetes a largo plazo. Con los estudio disponibles, no es posible establecer si la educación del paciente es eficaz en la pro- moción de los autocuidados que, a largo plazo, sirvan para prevenir o retrasar las complicaciones derivabas de la diabetes o una la mejora de la calidad de vida del paciente 5 .

Los resultados de la revisión sistemática de Vermeire $^{13}$, referente a ensayos clínicos aleatorizados, para la prevención de ulceras y el cumplimiento de las recomendaciones del tratamiento presenta alto grado de deficiencia metodológica, debido al sesgo de selección de la muestra; que es poco representativa, además de que los resultados obtenidos en su análisis no eran extrapolables a otros grupos de población. No obstante en cuanto a resultados de dichos estudios, se hallaron efectos significativos en la reducción de aparición de úlceras o amputación después de un seguimiento de aproximadamente un año y medio tras la intervención educativa, así mismo hubo una disminución del 10\% en los niveles de HbA1c en los tres primeros meses del estudio.

\section{DISCUSIÓN}

Ante los estudios que relacionan el autocuidado de los pacientes como prevención de complicaciones se observa se observa que las tres revisiones sistemáticas, que han evaluado la efectividad de intervenciones educativas en la prevención de lesiones de pie diabético, concluyen que existe presencia de déficit metodológi$\mathrm{co}^{13}$. Y que éste, puede deberse, en muchos casos, a la falta de aleatorización de la muestra o bien, a que no estén bien definidos los criterios de inclusión y exclusión ${ }^{14}$. Por lo que se hace necesario, mejorar las investigaciones que determinen estrategias de grado de cumplimiento de la práctica de buenos hábitos, para mejorar las complicaciones resultantes de la diabetes.

Por otro lado, nos enfrentamos también a la problemática planteada por el estudio realizado de 2008 realizado en Alemania que demostraba que la población de estudio, (pacientes alemanes con diagnóstico de diabetes) no estaba recibiendo suficiente información acerca de cómo realizar el autocuidado ${ }^{15}$.

De hecho, existe poca información específica, basada en la evidencia científica, a la hora de asesorar sobre el cuidado de los pies diabéticos ${ }^{16}$. Pues, en la actualidad, sólo existen dos 


\begin{tabular}{|c|c|c|c|}
\hline Estudio & Muestra & $\begin{array}{l}\text { Diseño e } \\
\text { intervención }\end{array}$ & Resultados \\
\hline Hinchliffe $2008^{1}$ & Pacientes isquémicos & $\begin{array}{l}\text { Revisión sistemática } \\
\text { (6 ECA) }\end{array}$ & $\begin{array}{l}\text { Educar a los pa- } \\
\text { cientes en cuidado } \\
\text { y observación de } \\
\text { los pies reduce las } \\
\text { úlceras }\end{array}$ \\
\hline Etoria $2009^{2}$ & DM tipo I y II & $\begin{array}{l}\text { Revisión sistemática } \\
\text { (14 ECA) }\end{array}$ & $\begin{array}{l}\text { Mejora de compli- } \\
\text { caciones con auto- } \\
\text { cuidado }\end{array}$ \\
\hline Vermeire $2008^{3}$ & DM tipo I y II & $\begin{array}{l}\text { Revisión sistemática } \\
\text { (9 ECA) }\end{array}$ & $\begin{array}{l}\text { Educar al paciente } \\
\text { puede reducir la } \\
\text { ulceración y las } \\
\text { amputaciones, } \\
\text { especialmente en } \\
\text { pacientes de alto } \\
\text { riesgo }\end{array}$ \\
\hline Canavan $2008^{24}$ & Pacientes amputados & Estudio prospectivo & $\begin{array}{l}\text { Disminución de } \\
\text { incidencia de ampu- } \\
\text { tación en pacientes } \\
\text { con cuidados de los } \\
\text { pies }\end{array}$ \\
\hline Johannes $2010^{14}$ & DM tipo I y II & $\begin{array}{l}\text { Revisión sistemática } \\
19 \text { estudios } 5 \text { (ECA) }\end{array}$ & $\begin{array}{l}\text { Sin pruebas su- } \\
\text { ficientes de que } \\
\text { las intervenciones } \\
\text { complejas reduzcan } \\
\text { las úlceras o com- } \\
\text { plicaciones de pie } \\
\text { diabético }\end{array}$ \\
\hline McInnes $2011^{8}$ & $\begin{array}{l}\text { DM Tipo I y II Bajo } \\
\text { riesgo complicaciones }\end{array}$ & Estudio prospectivo & $\begin{array}{l}\text { Mejora de compli- } \\
\text { caciones con auto- } \\
\text { cuidado }\end{array}$ \\
\hline Clark $2008^{4}$ & DM tipo I y II & Revisión simple & $\begin{array}{l}\text { Mejora de compli- } \\
\text { caciones con auto- } \\
\text { cuidado }\end{array}$ \\
\hline Deakin et al. ${ }^{27}$ & DM tipo II & $\begin{array}{l}\text { Revisión Cochrane } \\
\text { (11 estudios) }\end{array}$ & $\begin{array}{l}\text { Mejora de co- } \\
\text { nocimiento } \\
\text { peso,tratamientos y } \\
\text { control glucémico }\end{array}$ \\
\hline Lorig $2008^{22}$ & DM tipo I y II & Estudio prospectivo & $\begin{array}{l}\text { Mejora de compli- } \\
\text { caciones con auto- } \\
\text { cuidado }\end{array}$ \\
\hline
\end{tabular}

Tabla 1. Contenidos sobre autocuidado 
directrices específicas para esta población, la publicada por la American Diabetes Association (ADA) y la del Grupo de Trabajo Internacional sobre el pie diabético ${ }^{6,8}$.

A esto se une, que aunque se pone gran énfasis en la educación, en la prevención y el cuidado de los pies, no se ha conseguido demostrar de un modo claro la eficacia de la educación en la reducción de la incidencia de úlceras, como indica alguna revisión sistemática ${ }^{13}$ y algún artículo de revisión ${ }^{9}$.

Sin embargo, son diversas las investigaciones que determinan que mediante la intervención educativa se previene la aparición de úlcera por la reducción del impacto de los factores de riesgo desencadenantes expuestos con anterioridad, como son la neuropatía, la isquemia y la infección. Haciendo especial hincapié en evitar daños accidentales, mediante la adopción de conductas preventivas como: el control de la temperatura del agua del baño, evitar caminar descalzo y la promoción de la salud del pie mediante el lavado, secado, hidratación diaria y el autoexamen; lo que se denominaría autocuidados ${ }^{17}$.

Este mismo estudio muestra, también, aparente mejora en el comportamiento relacionado con el cuidado de los pies. Las diferencias observadas consistieron en obtención de puntuaciones más altas en ciertos elementos del cuidado de los pies, como la comprobación de los zapatos antes de ponérselos y el lavado y uso de crema hidratante a diario ${ }^{17}$.

En la misma línea, hallamos el estudio de Gabbay cuya intervención fue una evaluación minuciosa del riesgo de úlcera mediante revisiones semanales, con asesoramiento en autocuidado, cuidados podológicos y consejos acerca del uso de calcetines y calzado adecuado, para los pacientes con alto riesgo de ulceración. Se realizaron, por tanto, acciones encaminadas hacia un mayor grado de cumplimiento del autocuidado ${ }^{7}$.

A lo largo de los años, se ha trabajado en la idea de que la supervisión crónica de los pies de personas con diabetes, así como los programas educacionales, reducen la incidencia de las úlceras ${ }^{18,19}$.

En general, se cree que los pacientes diabéticos, especialmente aquellos con alto riesgo de ulceración del pie, deben aprender los principios del autoexamen y cuidado de los pies $^{20}$.

De hecho, en el año 2008 Ramon-Cabot ${ }^{21}$ postuló llevar a cabo un programa de prevención y reducción de úlceras en población diabética. que, hacía referencia a los cuidados básicos de higiene, calzado adecuado y autocuidado del paciente. Su objetivo era la reducción de complicaciones derivadas de una mala práctica o ausencia de hábitos saludables ${ }^{21}$.

Así mismo, los estudios publicados por Lincoln y Lorig en el año 2008 concluyen en la necesidad de ampliar los programas de prevención para la reducción de complicaciones derivadas de la diabetes, especialmente la aparición de úlceras ${ }^{17,22}$.

Sin embargo, estos datos deben ser observados con cautela si tenemos en cuenta que el autocuidado adecuado del pie no lo realizan la mayoría de los pacientes con diabetes ${ }^{23}$.

Además, en base a la experiencia de nuestras consultas, encontramos que existen diferencias en torno al tipo de paciente diabético, en cuanto a características socioeconómicas, grado de conocimiento, control de la enfermedad y desarrollo de buenos hábitos de salud.

Hasta ahora, hemos visto que la mayoría de las intervenciones para mejorar las conductas de autocuidado se han centrado en aumentar el conocimiento, aunque el supuesto de que la transferencia de conocimientos mejora los resultados sanitarios, tiene poco apoyo empírico. Casi todos los estudios que han utilizado la educación en comparación con las intervenciones conductuales y su influencia en el comportamiento, son poco concluyentes. Se ha evaluado la efectividad de intervenciones educativas en la prevención de lesiones del pie diabético con la conclusión general de que presentan mala calidad metodológica ${ }^{14}$.

Por otro lado, un estudio concluye que el nivel de hemoglobina glicosilada media fue significativamente más bajo, después de la intervención mediante el adiestramiento en conductas de autocuidado. Esta diferencia en el control de la glucemia se mantuvo a los 24 me$\mathrm{ses}^{25}$. Trabajando con pacientes de alto riesgo de complicaciones diabéticas, el autocuidado es fundamental para reducir las tasas de úlcera y amputación. Siendo un factor determinante de los resultados, el pobre autocuidado que los 
pacientes dedican a sus pies; por lo que los profesionales sanitarios tienen obligación de ayudar a orientar a los pacientes hacia una mejora del autocuidado ${ }^{7}$.

Sin embargo, Vermeire ${ }^{13}$ señala que existe relación entre la educación al paciente diabético y la reducción de úlceras y amputaciones. De hecho uno de los objetivos de la declaración de Saint Vincent de 1989 fue la reducción del 50\% de las amputaciones derivadas de la diabetes, en los cinco años posteriores ${ }^{5}$ así como el hecho de la falta de conocimiento de enfermos y profesionales, junto con un inadecuado tratamiento; que están dando lugar a una morbilidad innecesaria.

Desde el punto de vista de la presencia de amputaciones y desarrollo de ulceración en miembros inferiores en población diabética y no diabética, Canavan ${ }^{24}$, habla en 2008 de que el riesgo relativo de amputación es 46 veces mayor a cinco años vista, en población diabética respecto de la no diabética, de ahí la importancia de la prevención de complicaciones en este grupo de población.

En cuanto a la microangiopatía y la neuropatía, en Alemania, en el año 2008 nos encontramos con un estudio que valora, la modificación de conductas en relación al benificio que aporta para esta complicación y su relación con la calidad de vida relacionada con la salud ${ }^{26}$.

Por otro lado, La revisión relativa a intervenciones complejas para la prevención de úlceras de pie diabético del año 2010 concluye que "si se informan los cambios en el conocimiento del paciente sobre el cuidado de los pies y su comportamiento de autocuidado, estos cambios deben medirse con herramientas estandarizadas y validadas"14 en los que se concluyó que las evaluaciones de educación han tenido una calidad variable, pero frecuentemente de- ficiente y han sido propensas a sesgo de selección y medición.

Sin embargo, la mayoría de autores señalan que el conocimiento y las habilidades son necesarias, pero no suficientes, para asegurar un buen control de la diabetes a largo plazo. Con los estudios disponibles, no es posible establecer si la educación del paciente es eficaz en la promoción de los autocuidados que, a largo plazo, sirvan para prevenir o retrasar las complicaciones derivabas de la diabetes o a la mejora de la calidad de vida del paciente ${ }^{5}$.

\section{LIMITACIONES}

En cuanto a las limitaciones impuestas por la investigación, algunas de las referencias consultadas presentan, a juicio de los autores, una deficiencia importante en la selección de la muestra o en la metodología, en particular, al definir el objetivo de la investigación, ya que esta se centró en el análisis de la calidad de vida en relación con la salud en lugar de evaluar la aparición de complicaciones en los pies que sufren las personas con diabetes ligados a su autocuidado y autoexamen, por ejemplo.

\section{CONCLUSIONES}

A la vista de los resultados, casi toda la literatura específica, se concluye que mejorar conductas como los hábitos de higiene, la hidratación, el calzado, el tipo de calcetines y el cuidado podológico, contribuyen positivamente a evitar posibles complicaciones. Sin embargo, sólo dos revisiones fueron sistemáticas y la mayoría analizaron estudios no controlados, por lo que sus resultados deben considerarse con cautela.

\section{BIBLIOGRAFÍA}

1. Hinchliffe RJ, Valk GD, Apelqvist. A systematic review of the effectiveness of interventions to enhance the healing of chronic ulcers of the foot in diabetes. Diabetes Metab Res Rev .2008. 24(1):119144

2. Chin YF, Huang TT.Development and validation of a diabetes foot self-care behavior scale. J Nurs Res. 2013 Mar;21(1):19-25.

3. Etoria-King H, Sinha S, Scammell A. Self-care of the diabetic foot: a literature review. Diabetic Foot Journal, $2009 ; 12(1)$

4. Clark M.Diabetes self-management education: A review of published studies. Primary care diabetes 


\section{2008. $113-120$}

5. World Health Organisation (Europe) and International Diabetes Federation (Europe). Diabetes Care and Research in Europe:The St Vincent Declaration. Diabet Med. 1990; 7:360

6. American Diabetes Association. Standards of medical care in diabetes-2009. Diabetes Care 2009; 32: S13-61.

7. Gabbay RA, Kaul S, Ulbrecht J, Scheffler NM, Armstrong DG. Motivational interviewing by podiatric physicians: a method for improving patient self-care of the diabetic foot. J Am Podiatr Med Assoc. 2011 Jan-Feb;101(1):78-84

8. McInnes A. No consensus between HCPs on diabetic foot care education. Diabetic Foot 2010; 13: 29-38.

9. McInnes A, Jeffcoate W, Vileikyte L, Game F, Lucas K, Higson N, Stuart L, Church A, Scanlan J, Anders J. Foot care education in patients with diabetes at low risk of complications: a consensus statement. Diabet Med. 2011 Feb;28(2):162-7.

10. Lázaro Martínez JL, Aragón Sánchez FJ, Beneit Montesinos JV, González Jurado MA, García Morales E, Martínez Hernández D. Foot biomechanics in patients with diabetes mellitus: doubts regarding the relationship between neuropathy, foot motion, and deformities. J Am Podiatr Med Assoc. 2011 May-Jun;101(3):208-14

11. International Working Group on the Diabetic Foot (IWGDF). International Consensus on the Diabetic Foot \& Practical Guidelines on the Management and Prevention of the Diabetic Foot 2007 [Interactive versión on DVD]. IWGDF-Consultative Section of the IDF (International Diabetes Federation). Compatible con Windows 98 or later

12. CASPe | Critical Appraisal Skills Programme Español [http://www.redcaspe.org/drupal/]

13. Vermeire E, Wens J, Van Royen P, Biot Y, Hearnshaw H, Lindenmeyer A. Intervenciones para mejorar el cumplimiento de las recomendaciones de tratamiento en personas con diabetes mellitus tipo 2 (Revisión Cochrane traducida).2. The Cochrane Library, 2008; 2

14. Johannes A N Dorresteijn, Didi M W Kriegsman, Gerlof D Valk. Intervenciones complejas para la prevención de la úlcera del pie diabético. Cochrane Database Syst Rev.2010; 1(1): CD007610

15. Schmidt S, Mayer H, Panfil EM. Diabetes foot self-care practices in the German population. J Clin Nurs. 2008 Nov;17(21):2920-6.

16. Harvey JN, Lawson VL. The importance of health belief models in determining self-care behaviour in diabetes. Diabet Med 2009; 26: 5-13.

17. Lincoln NB, Radford KA, Game FL, Jeffcoate WJ. Education for secondary prevention of foot ulcers in people with diabetes: a randomised controlled trial. Diabetologia. 2008; 51:1954-61

18. Salomé GM, Maria de Souza Pellegrino D, Blanes L, Ferreira LM. Self-esteem in patients with diabetes mellitus and foot ulcers. J Tissue Viability. 2011 Aug;20(3):100-6.

19. Pinilla AE, Sánchez AL, Mejía A, Barrera Mdel P. Primary-care prevention activities in outpatients suffering from diabetic foot care. Rev Salud Publica (Bogota). 2011 Apr;13(2):262-73.

20. Perrin BM, Swerissen H, Payne C. The association between footcare self efficacy beliefs and actual foot-care behaviour in people with peripheral neuropathy: a cross-sectional study. J Foot Ankle Res 2009; $2: 3$

21. Ramon-Cabot J, Fernández-Trujillo M, Forcada-Vega C, Pera-Blanco G. Medium-term effectiveness of a group educational intervention on foot care in patients with type 2 diabetes. Enferm Clin. 2008 Nov-Dec;18(6):302-8.

22. Lorig K, Ritter PL, Villa F, Piette JD Spanish Diabetes Self-Management With and Without Automated Telephone Reinforcement Diabetes Care2008; 31:408-414

23. García-Morales E, Lázaro-Martínez JL, Martínez-Hernández D, Aragón-Sánchez J, Beneit-Montesinos J.V., and González-Jurado M.A. Impact of Diabetic Foot Related Complications on the Health Related Quality of Life (HRQol) of Patients A Regional Study in Spain. The International Journal of Lower Extremity Wounds.2010.10 (1): 6-11

24. Canavan RJ, Uwin NC, Kelly WF, Connolly VM. Diabetes- and Nondiabetes-Related Lower Extremity Amputation Incidence Before and After the Introduction of Better Organized Diabetes Foot Care Diabetes Care. 2008; 31: 459-463

25. Happich M, John J, Stamenitis S, Clouth J, Polnau D (2008) The quality of life and economic burden of neuropathy in diabetic patients in Germany in 2002-results from the Diabetic Microvascular Complications (DIMICO) study. Diabetes Res Clin Pract 81:223-230

26. Ribu L, Birkeland K, Hanestad BR, Moum T, Rustoen T . A longitudinal study of patients with dia- 
betes and foot ulcers and their health-related quality of life: wound healing and quality-oflife changes. J Diabetes Complications.2008, 22:400-407

27. Deakin T, McShane CE, Cade JD, Williams RD, Group based training for self-management strategies in people with type 2 diabetes mellitus, Cochrane Database Syst. Rev. (2) (2005), doi:10.1002/14651858. CD003417. pub2, CD003417. 